\title{
Inhibition of PTTG1 expression by microRNA suppresses proliferation and induces apoptosis of malignant glioma cells
}

\author{
XING SU $^{1 *}$, JIANGUO CHEN ${ }^{1 *}$, LANCHUN NI $^{1}$, WEI SHI $^{1}$, JINLONG SHI $^{1}$, XIAOJIANG LIU ${ }^{1}$, \\ YU ZHANG ${ }^{1}$, PEIPEI GONG ${ }^{1}$, HUI ZHU ${ }^{2}$ and QINGFENG HUANG ${ }^{1}$ \\ ${ }^{1}$ Department of Neurosurgery; ${ }^{2}$ Comprehensive Surgical Laboratory, Affiliated Hospital of Nantong University, \\ Nantong, Jiangsu 226001, P.R. China
}

Received February 16, 2015; Accepted February 19, 2016

DOI: $10.3892 / 01.2016 .5035$

\begin{abstract}
The present study aimed to investigate the role of pituitary tumor-transforming gene 1 (PTTG1) in the proliferation, invasion and apoptosis of human malignant glioma U251 cells. Firstly, 2 microRNAs (miRNAs) targeting PTTG1 messenger (m)RNA were ligated into a pcDNA6.2-GW/EmGFP-miR expression vector. The recombinant plasmids, miRNA-1 and miRNA-2 (miR-2), were transfected into U251 cells using the liposome method. PTTG1 mRNA and protein levels were evaluated using quantitative polymerase chain reaction and western blot analysis. The proliferation and invasion abilities of U251 cells were determined using methylthiazol tetrazolium and Matrigel assays. Flow cytometry analysis with Annexin V/propidium iodide double staining was used to determine the percentage of apoptotic cells. PTTG1 expression was effectively suppressed by miR-2. U251 cell growth was inhibited between 10.7 and $34.7 \%$ in the miR-2 group compared with the blank group. The Matrigel assay demonstrated that the percentage of infiltrating U251 cells was significantly lower in the miR-2 group $(12.3 \pm 1.0 \%)$ compared to the blank group $(24.7 \pm 1.4 \% ; \mathrm{P}<0.001)$ and the negative control group $(24.0 \pm 2.0 \% ; \mathrm{P}<0.05)$. A higher percentage of apoptotic U251 cells were observed in the miR-2 group compared with the blank group (53.6 vs. 32.4\%) using flow cytometry due to cycle arrests at the G2/M phase. The miR-2-transfected U251 cells were subcutaneously injected into nude mice, and these mice possessed a decreased tumor tissue growth rate
\end{abstract}

Correspondence to: Dr Qingfeng Huang, Department of Neurosurgery, Affiliated Hospital of Nantong University, 20 Xisi Road, Nantong, Jiangsu 226001, P.R. China

E-mail: hqf025@ntu.edu.cn

Mrs. Hui Zhu, Comprehensive Surgical Laboratory, Affiliated Hospital of Nantong University, 20 Xisi Road, Nantong, Jiangsu 226001, P.R. China

E-mail:237356779@qq.com

${ }^{*}$ Contributed equally

Key words: PTTG1, microRNAs, glioma, cell proliferation, tumor invasion, cell apoptosis and higher percentage of apoptotic cells compared with the blank and negative control groups. In conclusion, PTTG1 gene expression in human malignant glioma U251 cells was effectively suppressed by exogenous miR-2. The downregulation of PTTG1 induced glioma cell apoptosis and cell cycle arrest at the $\mathrm{G} 2 / \mathrm{M}$ phase, which inhibited cell proliferation, reverse invasion and infiltration of glioma cells.

\section{Introduction}

The pituitary tumor-transforming gene 1 (PTTG1) is an oncogene that was originally identified in the mouse pituitary tumor GH4 cell line using a messenger (m)RNA differential display polymerase chain reaction (PCR) technique (1). PTTG1 has been reported to be highly expressed in malignant astrocytoma and is closely associated with tumor invasion (2). As a potent tumor transforming gene, PTTG1 induces oncogenesis by facilitating cell proliferation and independent tumorigenesis in in vivo and in vitro studies $(3,4)$. However, the complex mechanism by which PTTG1 affects tumor cell proliferation and invasion remains unclear and requires additional investigation. It has been demonstrated that PTTG1 regulates cell proliferation via a mitogen-activated protein kinase (MAPK) phosphorylation site (proline-X-serine/threonine-proline) in its transcriptional activation domain, which using serine162 as the specific site. This is important for the PTTG1 transcription activation that is potentiated by the MAPK signal pathway and involves various growth factors, including epidermal growth factor (5-7). Previous studies using human cervical adenocarcinoma HeLa S3 cells demonstrated that the c-Myc gene acts as a downstream target of PTTG1 in tumorigenesis, accompanied by an upregulation in cell proliferation and colony formation, following the induction of PTTG1 expression $(8,9)$. Overall, the MAPK and c-Myc pathways may be involved in PTTG1-induced cell proliferation, although additional studies are required to confirm this hypothesis.

MicroRNAs (miRNAs) are endogenous non-coding RNAs, 20-23 nucleotides long, which negatively regulate gene expression at the transcriptional level by complementary base paring with their target mRNAs to induce mRNA degradation or translation inhibition (10). It has been demonstrated that miRNAs are associated with oncogenesis. Levels of certain miRNAs are reduced in several human cancers, suggesting the 
potential function of miRNAs as tumor inhibitors under normal conditions (11). miRNAs regulate gene expression by inhibiting target protein synthesis, as reported by Lewis et al (12), who developed a computational model to identify the target genes of miRNAs and revealed that miRNAs are involved in numerous biological functions. These findings suggest potential applications of miRNAs as drug candidates in cancer treatment, as specific gene expression may be blocked by RNA interference using synthetic miRNAs, which has potential prospects in treating various cancers and genetic diseases, such as colon, breast and lung cancer, hepatocellular carcinoma, Parkinson's disease, Alzheimer's disease and Huntington's disease. To provide an improved understanding of the effect of PTTG1 on the proliferation and invasion of human glioma cells, the present study suppressed the expression of the PTTG1 gene using exogenous miRNA induced by pcDNA6.2-GW/EmGFP-miR. In addition, the present study investigated the role of PTTG1 in inducing the apoptosis of human malignant glioma U251 cells.

\section{Materials and methods}

Sample preparation and hematoxylin and eosin (HE) staining. The 52 samples of glioma tissues used were obtained from surgical resections performed between 2012 and 2014 at the Affiliated Hospital of Nantong University (Nantong, China). All fresh frozen human glioma tissue samples were obtained and in accordance with an Institutional Review Board protocol approved by the Partners Human Research Committee. The tissues were rinsed in normal saline and divided into three sections. One section was fixed in $10 \%$ formalin (Boster Biological Technology, Ltd., Wuhan, China) for routine pathological examinations. The other two sections were frozen immediately in liquid nitrogen, stored at $-70^{\circ} \mathrm{C}$ and were used for immunohistochemical (IHC) staining and western blot analysis.

Following fixation, the tissues were embedded in paraffin and sectioned. All the tissue slices were dewaxed in xylene (Chengdu Feike BioTechnology Co., Ltd, Chengdu, China), rehydrated in gradient ethanol $(35,55,75$ and $95 \%$ ethanol; Chengdu Feike BioTechnology Co., Ltd), stained in hematoxylin (Chengdu Feike BioTechnology Co., Ltd) for $5 \mathrm{~min}$, washed in tap water, dehydrated in gradient ethanol and sealed in neutral gum. The tissue morphology was observed under a light-field microscope (BX50F; Olympus, Tokyo, Japan).

IHC staining. Human malignant glioma U251 cells were provided by the Chinese Academy of Science (Shanghai, China) and were cultured in Gibco Dulbecco's modified Eagle's medium (DMEM; Thermo Fisher Scientific, Inc., Waltham, MA, USA) supplemented with $10 \%$ fetal bovine serum (FBS; Jackson ImmunoResearch Laboratories, Inc., West Grove, PA, USA) at $37^{\circ} \mathrm{C}$ in a $5 \% \mathrm{CO}_{2}$ atmosphere. The cells were passed with $0.25 \%$ trypsin (Boster Biological Technology, Ltd.) at a split ratio of 1:3. U251 cells in the log phase of growth were collected and rinsed in sterilized phosphate-buffered saline (PBS; PAA Laboratories GmbH, Pasching, Austria) three times and fixed with $2 \mathrm{ml}$ sterilized $4 \%$ paraformaldehyde (Chengdu Feike BioTechnology Co., Ltd) for $30 \mathrm{~min}$. Following three washes with PBS, $3 \% \mathrm{H}_{2} \mathrm{O}_{2}$ (Dakewe Biotech
Co., Ltd, Shenzhen, China) was used to quench the endogenous peroxidase activity. A rabbit anti-PTTG1 polyclonal antibody (dilution, 1:500; catalog no., 34-1500; Invitrogen; Thermo Fisher Scientific, Inc.) was incubated with cells at $4^{\circ} \mathrm{C}$ overnight subsequent to $1 \mathrm{~h}$ blocking in 5\% goat serum (PAA Laboratories $\mathrm{GmbH}$ ) containing $0.1 \%$ Triton X (Carl Roth GmbH \& Co. KG, Karlsruhe, Germany). A negative control was performed using PBS instead of the primary antibody. A mouse anti-rabbit horseradish peroxidase (HRP)-conjugated secondary antibody (dilution, 1:200; catalog no., sc-2004; Invitrogen; Thermo Fisher Scientific, Inc.) was used to amplify the signal with the chromogenic substrate 3,3'-diaminobenzidine (Invitrogen; Thermo Fisher Scientific, Inc.). Using a light-field microscope equipped with a measurement grid, 500-1,000 cells were counted from 10 randomly selected fields under a x400 magnification.

Vector constructs and cell transfection. The Invitrogen pcDNA6.2-GW/EmGFP-miR plasmids were purchased from Thermo Fisher Scientific, Inc. Competent Escherichia coli cells were purchased from Tiangen Biotech Co., Ltd. (Beijing, China). Two pairs of miRNA sequences (Table I) were designed using Invitrogen Block-iT ${ }^{\text {тм }}$ RNAi Designer software (Thermo Fisher Scientific, Inc.) based on the human PTTG1 gene sequence (gene ID, 3232; GeneBank; National Center for Biotech Information, Bethesda, MD, USA). Each oligonucleotide possessed a length of $64 \mathrm{bp}$ and could form a hairpin structure. These oligonucleotides were able to form unique sticky ends following annealing, and could be ligated onto the sticky ends of the pcDNA6.2-GW/EmGFPmiR vector. The oligonucleotides $(10 \mu \mathrm{M})$ were incubated in Invitrogen annealing buffer (Thermo Fisher Scientific, Inc.) at $95^{\circ} \mathrm{C}$ for $4 \mathrm{~min}$ and $37^{\circ} \mathrm{C}$ for $10 \mathrm{~min}$ prior to being ligated to the empty vector using Invitrogen T4 ligase (Thermo Fisher Scientific, Inc.). Recombinant vectors were used to transform competent cells, which were then seeded onto lysogeny broth (LB)-spectinomycin culture plates (BD Biosciences, Franklin Lakes, NJ, USA). In total, 4 monoclonal colonies were additionally incubated in $3 \mathrm{ml}$ of LB medium (Sigma-Aldrich, St. Louis, MO, USA) containing $50 \mu \mathrm{g} / \mathrm{ml}$ of cells at $37^{\circ} \mathrm{C}$ overnight. Extracted plasmids were screened by a double digestion with $B s r \mathrm{DI}$ and BamHI (Invitrogen; Thermo Fisher Scientific, Inc.) and confirmed by sequencing (reverse, 5'-CTCTAGATCAACCACTTTGT-3'; forward, 5'-ACA ATCAGATTACTAACGAG-3'; Invitrogen, Thermo Fisher Scientific, Inc.). The results revealed that the recombinant pcDNA6.2-GW/EmGFP-miR-PTTG1 plasmid had an inserted target gene of $64 \mathrm{bp}$, which was the same as the original design. Positive constructs with the correct miRNA insertions were termed miRNA-1 (miR-1) and miRNA-2 (miR-2), with the negative control being termed Neg. All plasmids were purified using the AxyPrep kit (Corning Inc., Corning, NY, USA) for additional transfection.

U251 cells in the log phase of growth $\left(1 \times 10^{5}\right.$ cells $\left./ \mathrm{ml}\right)$ were digested, seeded into 6-well plates (BD Biosciences) and cultured for $48 \mathrm{~h}$ to reach a stable growth status with $80 \%$ confluency. miR-1, miR-2 and Neg plasmids were transfected into U251 cells using Invitrogen Lipofectamine ${ }^{\circledR} 2000$ (Thermo Fisher Scientific, Inc.) following the manufacturer's protocol. An empty control group was established by adding 
Lipofectamine 2000 only. The transfection efficiency was determined subsequent to $24 \mathrm{~h}$ by evaluating emerald green fluorescent protein (EmGFP) expression under an Axiovert 200M inverted fluorescence microscope (excitation, 488 nm; emission, 509 nm; Carl Zeiss, AG, Oberkochen, Germany).

Quantitative PCR ( $P$ PCR) and western blot analysis. Total RNA was extracted from U251 cells $48 \mathrm{~h}$ subsequent to transfection using RNeasy Mini kit (Qiagen China Co., Ltd., Shanghai, China) and was measured for PTTG1 mRNA levels using qPCR. Primer sequences for PTTG1 and glyceraldehyde 3-phosphate dehydrogenase, as an internal control, are reported in Table I. qPCR was performed by quantitative fluorescent PCR reactor (BioRad Laboratories, Inc., Hercules, CA, USA) using the following program repeated for 40 cycles: $94^{\circ} \mathrm{C}$ for $4 \mathrm{~min} ; 94^{\circ} \mathrm{C}$ for $20 \mathrm{sec}$; and $60^{\circ} \mathrm{C}$ for $30 \mathrm{sec}$. All samples were normalized to $\beta$-actin using the $2^{-\Delta \Delta \mathrm{Cq}}$ method (13).

The cells were lysed $72 \mathrm{~h}$ subsequent to transfection with 2-D lysis buffer (Beyotime Institute of Biotechnology, Haimen, China) and the total protein was quantified using the Bradford method. The cell lysates were subjected to $12 \%$ sodium dodecyl sulfate-polyacrylamide gel electrophoresis separation, transferred to polyvinylidene difluoride membranes (Merck Millipore, Darmstadt, Germany), blocked in 5\% non-fat milk powder (Sangon Biotech, Shanghai, China) and incubated with rabbit anti-human PTTG1 polyclonal antibody (dilution, 1:500), mouse anti-Ki67 monoclonal antibody (dilution, 1:100; catalog no., sc-15402; Santa Cruz Biotechnology, Inc., Dallas, TX, USA), mouse anti-matrix metalloproteinase (MMP)-2 monoclonal antibody (dilution, 1:100; catalog no., MS-567-P0; Thermo Fisher Scientific, Inc.), mouse anti-MMP-9 monoclonal antibody (dilution, 1:100; Thermo Fisher Scientific, Inc.) or Invitrogen mouse anti-human $\beta$-actin (dilution, 1:500; catalog no., sc-2405; Thermo Fisher Scientific, Inc.). Subsequent to $2 \mathrm{~h}$ incubation at room temperature and washing with Tris-buffered saline and Tween 20 (Beyotime Institute of Biotechnology), HRP-conjugated goat anti-rabbit immunoglobulin G secondary antibodies (dilution, 1:400; Santa Cruz Biotechnology, Inc.) were added to the membrane for a $1 \mathrm{~h}$ incubation, followed by rinsing with Tris-buffered saline, and developed using enhanced chemiluminescence reagent (Pierce Biotechnology, Inc., Rockford, IL, USA) and $\mathrm{X}$-ray exposure. Western blot analysis bands were quantified using the Odyssey densitometry program (LI-COR Biosciences, Lincoln, NE, USA.). $\beta$-actin was used as an internal control for normalizing the protein load.

Cell proliferation and migration assay. The cells in the log phase of growth were seeded in 96 -well plates $\left(1 \times 10^{5}\right.$ cells $/ \mathrm{ml}$; $100 \mu \mathrm{l} /$ well; BD Biosciences) and cultured at $37^{\circ} \mathrm{C}$ with $5 \%$ $\mathrm{CO}_{2}$ for $18 \mathrm{~h}$. The cells were washed with Gibco Opti-MEM ${ }^{\mathrm{TM}}$ (Thermo Fisher Scientific, Inc.) without serum and transfected with control and miRNA plasmids. In total, $100 \mu 1$ fresh Opti-MEM medium (Gibco; Thermo Fisher Scientific, Inc.) was added $6 \mathrm{~h}$ subsequent to transfection. Methylthiazol tetrazolium (MTT) reagent $(100 \mu \mathrm{l} /$ well) was added $4 \mathrm{~h}$ prior to each time point. The cells were then treated with $100 \mu 1$ dimethyl sulfoxide (Boster Biological Technology, Ltd.) and were agitated for 15 min until all the crystals had been dissolved. The optical density absorption values were measured at $490 \mathrm{~nm}$ and the mean values were calculated from 6 repeated experiments.

Matrigel (BD Biosciences) was thawed overnight at $4^{\circ} \mathrm{C}$, diluted with DMEM without serum and added to the top chamber of a pre-cold Transwell filter plate (pore size, $8 \mu \mathrm{m}$; Costar; Cambridge, NY, USA). The 24-well plate was incubated for $2 \mathrm{~h}$ at $37^{\circ} \mathrm{C}$ to allow complete polymerization of the Matrigel. Transfected cells $\left(1 \times 10^{5}\right.$ cells; $100 \mu \mathrm{l} /$ well $)$ were added to the top chamber, while $600 \mu \mathrm{l}$ chemokines prepared from mouse embryo fibroblast NIH/3T3 cells (American Type Culture Collection, Manassas, VA, USA) (14) were added to the bottom chamber. The Transwell plate was incubated at $37^{\circ} \mathrm{C}$ with $5 \% \mathrm{CO}_{2}$ for $36 \mathrm{~h}$. The plate was cleaned to remove immobile cells, and was fixed in $100 \%$ methanol (Seebio Biotech, Inc., Shanghai, China) for $10 \mathrm{~min}$, followed by Giemsa staining (Seebio Biotech, Inc.) for $15 \mathrm{~min}$. The number of cells in 5 randomly selected fields from the lower region of the plate was counted under the light-field microscope at a magnification of $x 320$. The invasive ability of the tumor cells was determined by the relative number of invasive cells.

Following digestion, cultured cells $\left(10^{6}\right.$ cells/well) were seeded into 6-well plates with $10 \%$ FBS/DMEM medium for overnight incubation. The following day, vertical scratches were labeled inside each well using a 200- $\mu$ l micropipette tip (Shanghai Rong Tai Biochemical Engineering Co., Ltd., Shanghai, China). Following two washes with Hanks' solution (Seebio Biotech, Inc.), Opti-MEM I Reduced Serum medium (Thermo Fisher Scientific, Inc.) without serum was used to incubate cells for $24 \mathrm{~h}$. The total number of migrated cells in 5 randomly selected fields was counted using an inverted phase contrast microscope (Leica DMI3000 B; Leica, Wetzlar, Germany) at a magnification of x 100 .

Apoptosis assay. miRNA and Neg (200 nmol/l) constructs were used to transfect cells, which were incubated for $48 \mathrm{~h}$, prepared for cell slides, fixed in paraformaldehyde for $1 \mathrm{~h}$, washed with PBS 3 times, stained in the dark using $10 \mathrm{mg} / \mathrm{l}$ Hoechst 33258 (0.5 ml/well; Beyotime Institute of Biotechnology) and sealed in $500 \mathrm{~g} / 1 \mathrm{PBS}$ with glycerol (Beyotime Institute of Biotechnology). The cells were observed under a fluorescent microscope.

Assay of caspase-3 activity and the cell cycle. The cells were collected at various time points $(24,48,72$ and $96 \mathrm{~h})$ following transfection and centrifuged at $872 \mathrm{x}$ g for $5 \mathrm{~min}$ (Eppendorf 5415R; Eppendorf, Hamburg, Germany), re-suspended and counted to provide $1 \times 10^{6}$ cells $/ \mathrm{ml}$ cell suspensions. Subsequently, $0.3 \mathrm{ml}$ cell suspension from each group was mixed with $1 \mu \mathrm{l}$ Z-VAD-FMK (Santa Cruz Biotechnology, Inc.), incubated at $37^{\circ} \mathrm{C}$ with $5 \% \mathrm{CO}_{2}$ for $40 \mathrm{~min}$, centrifuged at $2616 \mathrm{x} \mathrm{g}$ for $5 \mathrm{~min}$ and was re-suspended in $5 \mathrm{ml}$ washing buffer for flow cytometry.

Subsequent to $48 \mathrm{~h}$ of transfection using $200 \mathrm{nmol} / \mathrm{l}$ miRNA, the cells were collected, digested using trypsin, centrifuged at $2616 \times \mathrm{g}$ and re-suspended in $5 \mathrm{ml}$ PBS. Following fixation in $70 \%$ ethanol at $4^{\circ} \mathrm{C}$ for $30 \mathrm{~min}$, the cells were stained using buffer containing RNase and propidium iodine (PI). The cell cycle analysis was performed using EPICS Elite ESP Cell Sorter (Beckman Coulter, Inc., Brea, CA, USA) with 
$\sim 11,000$ cells in each sample, via the percentage measurement of cell numbers at each phase.

Subcutaneous transplantation of tumors. The animal study proposal was approved by the Laboratory Animal Center of Nantong University (permit number, 20140310-009). All mouse experimental procedures were performed in accordance with the Regulations for the Administration of Affairs Concerning Experimental Animals approved by the State Council of People's Republic of China (15). In total, $21 \mathrm{BALB} / \mathrm{C}$ nude mice (4-6 weeks old; body weight, $18 \pm 2 \mathrm{~g}$ ) were provided by the animal experimental center at Affiliated Hospital of Nantong University (Nantong, Jiangsu, China) and kept in a specific-pathogen-free facility. The female mice were randomly divided into three groups: miR-2-transfected U251 cell group; Neg-U251 group; and non-transfected U251 (mock) group. Subsequent to $48 \mathrm{~h}$ of transfection, the cells were collected and digested, and a $5 \times 10^{7}$ cells $/ \mathrm{ml}$ cell suspension was rendered. In total, $0.2 \mathrm{ml}$ of the cell suspension was subcutaneously injected into the dorsal skin of the nude mice.

The tumor size was measured each week following inoculation. The tumor volume was calculated as follows: Tumor volume $=a b^{2} / 2$, where a was the length and $b$ was the width. The mice were sacrificed 4 weeks subsequent to inoculation. The tumor tissues were removed and weighed, and the differences between groups were compared. Sections of the tumor tissues were then fixed in $10 \%$ neutral-buffered formalin (Boster Biological Technology, Ltd.), embedded in paraffin (Boster Biological Technology, Ltd.) within $24 \mathrm{~h}$, sectioned into $4-\mu \mathrm{m}$ serial tissue slices and examined for tissue morphology. The remainder of the tumor tissues was frozen in liquid nitrogen for future use.

Statistical analysis. SPSS software version 11.0 (SPSS Inc., Chicago, IL, USA) was used to process all collected data, which were presented as the mean \pm standard deviation. The comparison of means was performed by one-way analysis of variance, $t$-test and $\chi^{2}$ test, as appropriate. Multiple comparisons between the groups were performed using the Student-Newman-Keuls method. $\mathrm{P}<0.05$ was considered to indicate a statistically significant difference.

\section{Results}

PTTG1 expression is significantly elevated in high-grade glioma. The present results from western blotting analysis (Fig. 1A) and fluorescent qPCR (Fig. 1B) revealed extremely low levels of PTTG1 in normal brain tissues. However, the protein expression level of PTTG1 became elevated with increased malignancy of glioma, as U251 cells possessed the most increased PTTG1 level.

IHC staining of the PTTG1 protein resulted in granular signals, and the tumor cell cytoplasm stained brown. In certain tumor samples, the peripheral connective tissues and vessel endothelial cells exhibited weak expression of PTTG1. The strength of staining was variable across tumor samples at various pathological stages, consisting of the fibrous astrocytoma, anaplastic astrocytoma and multiform glioblastoma stages. Tumors with a lower malignancy exhibited weak staining in astrocytes in comparison with advanced glioma, which demonstrated clear staining in the majority of cells (Fig. 1C).

Out of a total of 52 glioma samples, the PTTG1 gene was expressed in 39 samples, accounting for $75 \%$ of all glioma samples. By contrast, no PTTG1 was expressed in the healthy brain tissues. In the 39 samples that did express PTTG1, 11 tumor samples were classified as fibrous astrocytoma stage II (PTTG1 expression, 55\%), 11 samples were anaplastic astrocytoma stage III (PTTG1 expression, 55\%), and 17 samples were multiform glioblastoma stage IV (PTTG1 expression, 100\%), as demonstrated by Fig. 1D.

Suppression of PTTG1 expression by exogenous miRNA in human malignant glioma U251 cells. In total, $80 \%$ of the U251 cells were transfected with and expressed EmGFP $24 \mathrm{~h}$ subsequent to transfection. The qPCR results demonstrated that there was no significant difference in PTTG1 mRNA levels between the empty and Neg control groups $(\mathrm{F}=0.95$; $\mathrm{P}>0.05)$. However, the PTTG1 mRNA levels in the miR-1 and miR-2 groups were decreased compared with the empty group $(\mathrm{F}=258 ; \mathrm{P}<0.01)$.

Western blot analysis demonstrated that there were significantly lower PTTG1 protein levels in the miR-1 and miR-2 cells, compared with the empty and Neg control cells, while there was no difference in PTTG1 protein expression between the empty and Neg control groups.

Inhibition of glioma cell proliferation by PTTG1 suppression. The present study measured the light absorption value (A490) of the U251 cells using the MTT method at various time points $(24,48,72$ and $96 \mathrm{~h})$ following transfection. The present results demonstrated an increasing suppression of the A490 value in cells transfected with miR-2 subsequent to $24 \mathrm{~h}(\mathrm{P}<0.05$; Table II; Fig. 2A), suggesting the participation of PTTG1 in glioma cell proliferation.

In the western blot analysis, the expression of PTTG1, Ki67 and c-Myc in U251 cells (Fig. 2B and C) were all significantly decreased in cells transfected with miRNA-PTTG1 plasmids, but not in cells without transfection or with negative control plasmids.

PTTG1 suppression induces glioma cell apoptosis. The flow cytometry quantitative analysis revealed a statistically significant increase in the percentage of apoptotic cells in U251 cells transfected with miR-2 interference plasmids (apoptotic cells subsequent to $48 \mathrm{~h}, 53.6 \%$ ) compared with empty cells (apoptotic cells subsequent to $48 \mathrm{~h}, 32.4 \%$ ) and Neg cells (apoptotic cells subsequent to $48 \mathrm{~h}, 37 \%$; $\mathrm{F}=124 ; \mathrm{P}<0.001$; Fig. $3 \mathrm{~A}$ and $\mathrm{B}$ ). In addition, the present study measured the caspase-3 levels in the cells. The results demonstrated a significantly elevated caspase- 3 activity in miR-2-transfected U251 cells, compared with empty or Neg cells (Fig. 3C and D).

PTTG1 suppression inhibits glioma cell migration and invasion. In the scratch cell migration assay, the average migration distance of miR-2-transfected U251 cells (Fig. 4) on day 7 was $29.9 \mu \mathrm{m}$, which was significantly lower compared with empty cells $(58.03 \mu \mathrm{m})$ or Neg cells $(65.66 \mu \mathrm{m})$. On 

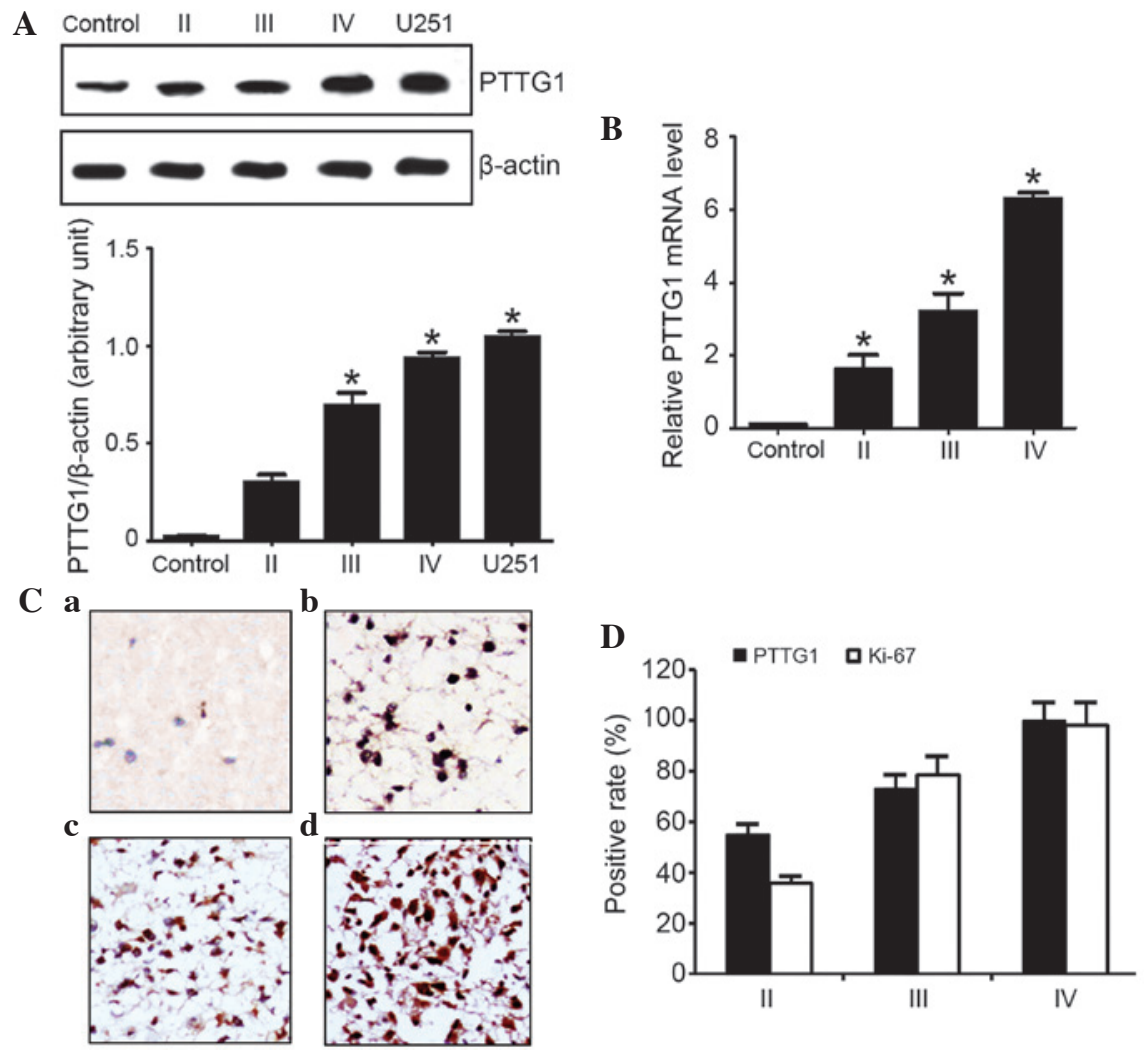

Figure 1. PTTG1 expression levels in glioma cells. (A) Western blot analysis and relative densities normalized against $\beta$-actin levels in glioma cells from stage II, III and IV glioma and U251 cells. (B) PTTG1 mRNA expression level in glioma cells. (C) Immunohistochemical staining using the anti-PTTG1 antibody in the following glioma tissues: (a) Control; (b) stage II glioma cells; (c) stage III glioma cells; and (d) stage IV glioma cells. (D) Quantification of the expression of PTTG1 and Ki-67 (positive rate) in cells from various stages of glioma. "P<0.05 vs. control group. PTTG1, pituitary tumor transforming gene 1; mRNA, messenger RNA.

A

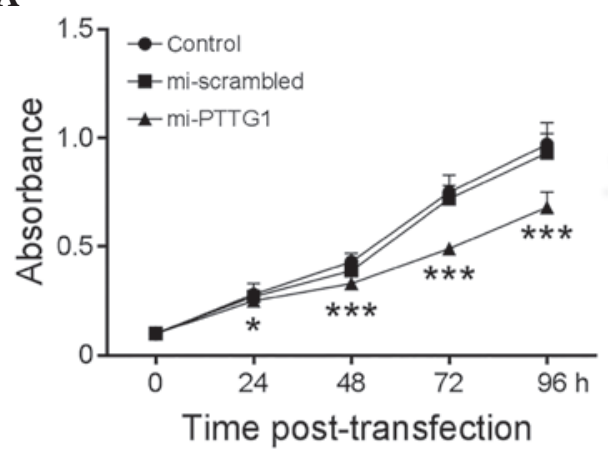

B
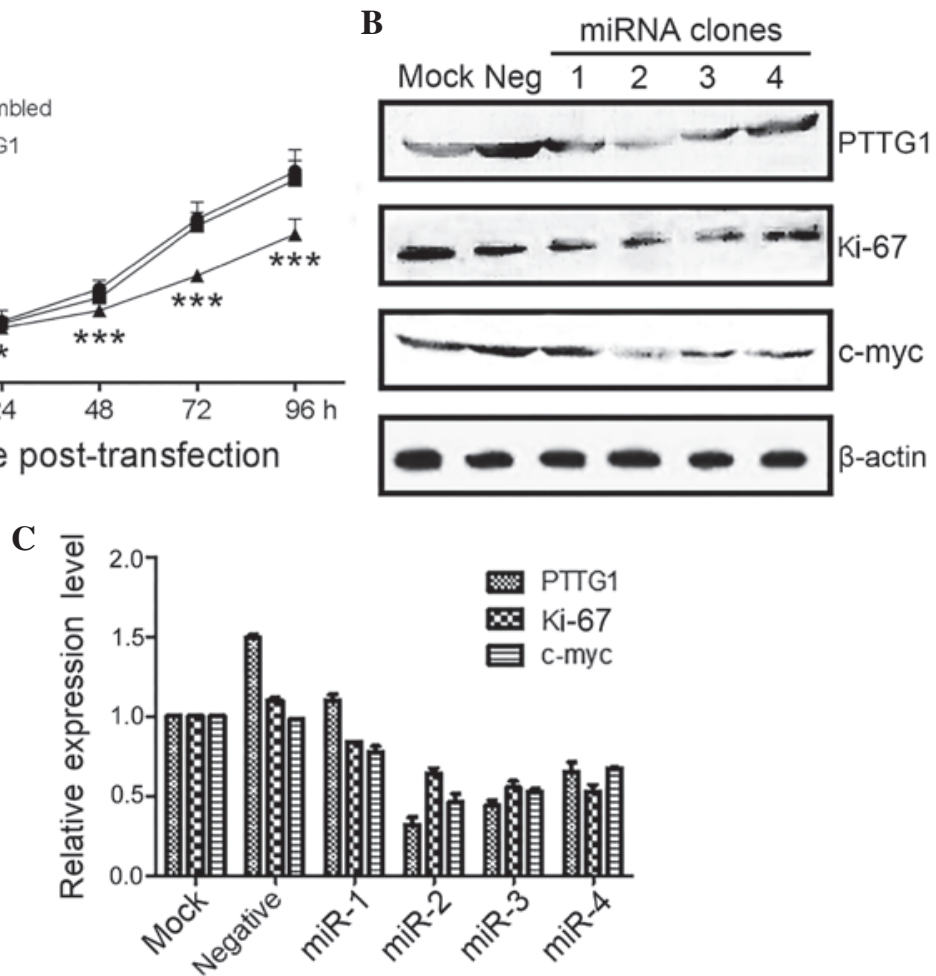

Figure 2. Cell growth and protein expression following PTTG1 suppression. (A) Cell growth absorption values were assessed using the methylthiazol tetrazolium method with human malignant glioma U251 cells in the mock, negative control and miR-2 groups. (B) Western blot analysis of various U251 cell groups exhibiting the expression of Ki67 and c-Myc proteins. (C) Quantitative results of Ki67 and c-Myc protein levels normalized against $\beta$-actin expression. * $<0.05$, ${ }^{* * * *} \mathrm{P}<0.001$ vs. control group. PTTG1, pituitary tumor transforming gene 1; miR, microRNA; mock, without transfection; Neg, empty plasmid transfection, miR-2, miR-2 plasmid transfection. 

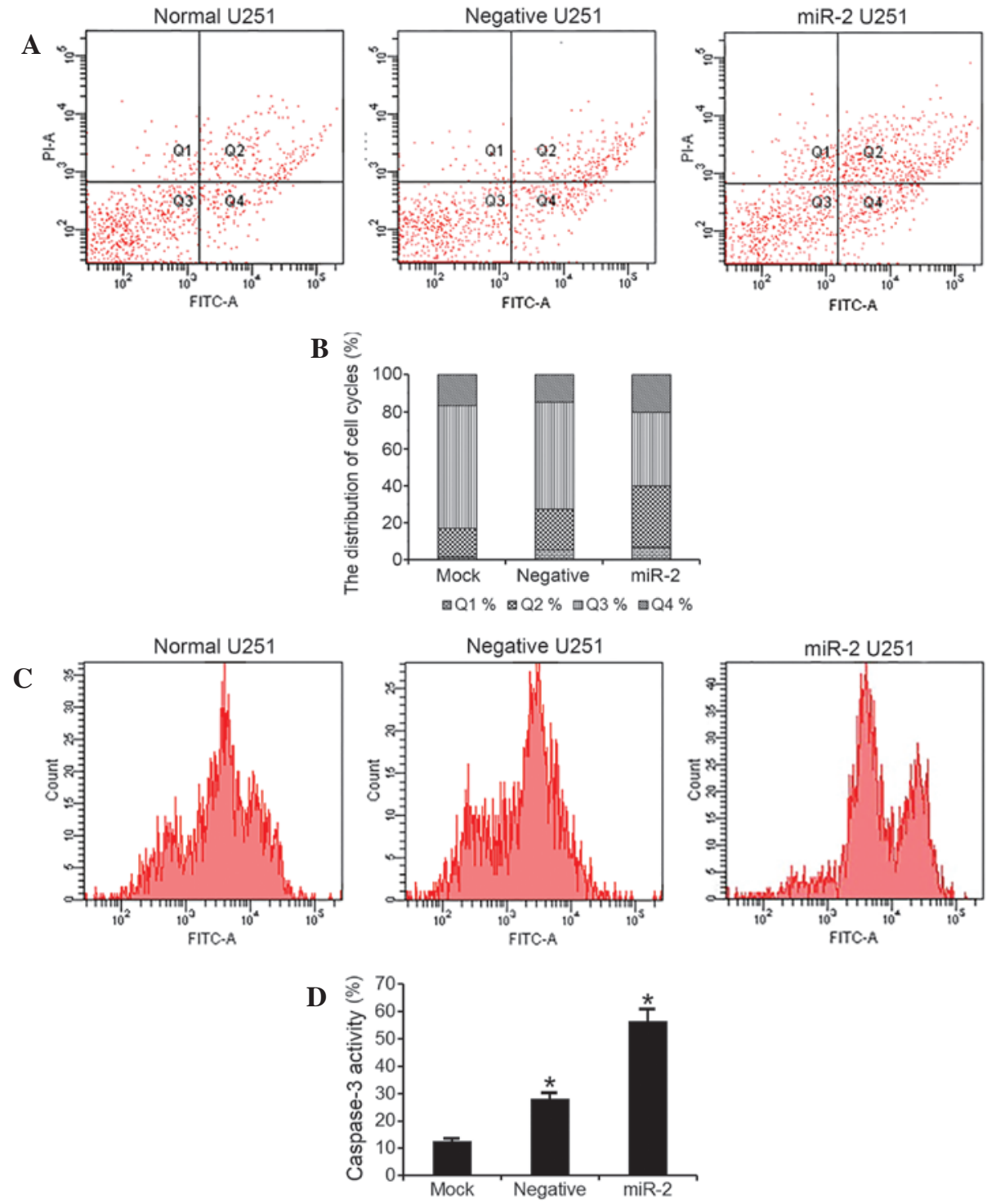

Figure 3. Cell apoptosis and caspase-3 activity. (A) Flow cytometry results obtained using the Annexin V/propidium iodide double staining method to detect cell apoptosis in normal, negative and miR-2-transfected U251 cells. (B) Quantitative results of flow cytometry. (C) Caspase-3 activity using flow cytometry analysis following transfection. (D) Quantitative results of caspase-3 activity using flow cytometry. ${ }^{*} \mathrm{P}<0.05$ vs. control (mock) group. miR, microRNA; U251, human malignant glioma U251 cell line; FITC, fluorescein isothiocyanate; Q1, errors; Q2, phase 2 apoptotic cells; Q3, normal cells; Q4, phase 1 apoptotic cells .

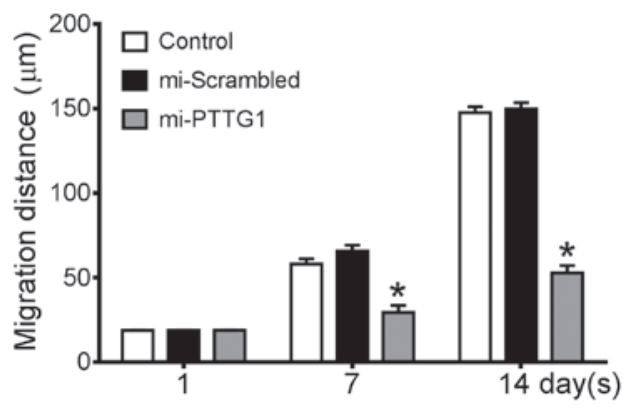

Figure 4. Cell migration was observed using the scratch method. The average migration distance of human malignant glioma U251 cells at 1,7 and 14 days following transfection. " $\mathrm{P}<0.05$ vs. control.mi, microRNA; PTTG1, pituitary tumor transforming gene 1 .

day 14, miR-2-transfected cells migrated by an average of $52.83 \mu \mathrm{m}$ compared with blank cells $(147.67 \mu \mathrm{m})$ or Neg cells (149.78 $\mu \mathrm{m})$.
The Matrigel invasion assay additionally revealed the impaired ability of glioma cell invasion when miRNA interference was present. The total number of invasive cells in 5 randomly selected fields at x100 magnification was 69.7 miR-2-transfected U251 cells, which was significantly decreased compared with empty (109.8 cells) or Neg cells (99.6 cells). The present study observed that there was a statistically significant decrease in the number of miR-2-transfected cells $(12.3 \pm 1.0 \%)$ that crossed the artificial basal membrane compared with the control cells $(24.7 \pm 1.4 \%$; $\mathrm{F}=266 ; \mathrm{P}<0.001)$.

In vivo studies of PTTG1 suppression-induced tumor growth inhibition. During the 4-week observation window, tumors in the mock mouse and Neg group exhibited rapid tumor growth, and the mice exhibited decreased activity, loss of body weight and a slow response. However, in the miR-2 group, the tumor exhibited a lower growth rate, and 


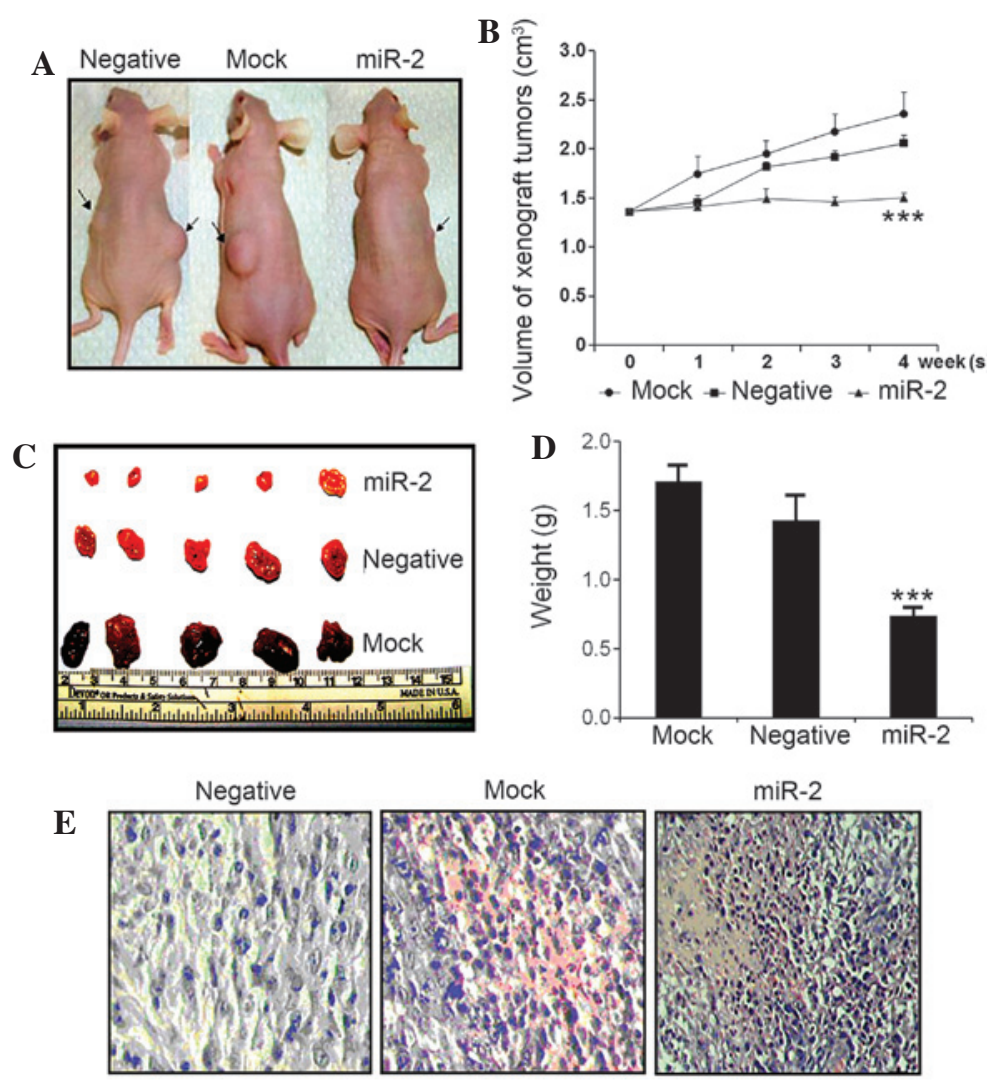

Figure 5. In vivo transplantation of glioma cells. (A) Mice transplanted with negative, normal and PTTG1-miRNA-transfected U251 cells. Arrows indicate tumor growth. (B) Growth curves of tumors between 0 and 4 weeks following transplantation, demonstrating the tumor volumes. (C) Tumors resected from sacrificed mice subsequent to 4 weeks. (D) Average weights of tumors. (E) Hematoxylin and eosin staining of resected tumors from mice inoculated with negative, normal and PTTG1-miRNA-transfected U251 cells. ${ }^{* * * *} \mathrm{P}<0.001$ vs. Mock animals. PTTG1, pituitary tumor transforming gene 1 miRNA, microRNA; miR-2, microRNA-2; U251, human malignant glioma U251 cell line; Mock, empty control.

the mice exhibited free activity and quick response rates. No mice in any group succumbed due to glioma or any other alternative causes.

In a longitudinal examination, tumor nodules formed $\sim 3$ days subsequent to the subcutaneous injection of glioma cells. Following 2 weeks, mock and Neg control mice, but not miR-2-transfected mice, possessed visible tumor nodules. Subsequent to 4 weeks, it was observed that mock mice had significantly larger tumors compared with the Neg and miR-2 groups (Fig. 5A). Following sacrifice, the tumors from the mock group were significantly larger in size (Fig. 5B; mock tumor size, $2.36 \pm 0.22 \mathrm{~cm}^{3}$; Neg tumor size, $1.82 \pm 0.15 \mathrm{~cm}^{3}$; miR-2 tumor size, $0.99 \pm 0.17 \mathrm{~cm}^{3}$ ) and weight (Fig. 5D; mock tumor weight, $1.71 \pm 0.13 \mathrm{~g}$; Neg tumor weight, $1.43 \pm 0.19 \mathrm{~g}$; miR-2 tumor weight, $0.74 \pm 0.07 \mathrm{~g}$ ) compared with tumors from the Neg and miR-2 groups $(\mathrm{P}<0.001)$. The tumor growth curve, which was plotted weekly and recorded tumor size, also demonstrated that the slow growth of tumors in mice injected with miR-2-transfected tumor cells was also significant (Fig. 5B).

In additional morphological examination of the tumor tissues using HE staining, tissue necrosis in the center of tumor was observed in mice injected with miR-2-transfected tumor cells, with sparsely distributed cell debris. The peripheral region of the tumor tissues also exhibited clear edema and hemorrhage and apoptotic features, including karyopyknosis (Fig. 5E).

\section{Discussion}

The present study successfully decreased PTTG1 expression levels in the human malignant glioma U251 cell line with exogenous PTTG1-miRNA. The proliferation of cells transfected with miRNA was significantly inhibited, accompanied by a slow growth of cells, and the cells possessed a lower transmembrane potential in a Matrigel assay. The present results support the hypothesis that glioma cells with a lower PTTG1 expression possess decreased invasive potential, suggesting that PTTG1 facilitates glioma invasion.

Tumor cells have an invasive nature, as they may penetrate the basal membrane of tissues and metastasize; therefore, evaluation of the migration ability of cells is an important index in the study of tumor cell invasion (16). The Matrigel cell migration assay performed in the present study reflected the process of tumor invasion and metastasis, including matrix degradation and cell adhesion and migration. In vitro studies have demonstrated that PTTG1 and basic fibroblast growth factor (b-FGF) may mutually induce the expression of each other and form feedback loops in an autocrine or paracrine manner $(17,18)$. PTTG1 expression and tumor invasiveness have been observed to be associated with the expression of $b-F G F$ and the b-FGF receptor FGF-R1, and also with vascular endothelial growth factor and its receptor kinase insert domain receptor in pituitary tumors (7). This demonstrates the close association between PTTG1 and 
Table I. Oligonucleotide sequences of miRs for reverse transcription-quantitative polymerase chain reaction.

\begin{tabular}{|c|c|c|c|c|c|}
\hline Primer name & \multicolumn{5}{|c|}{ Sequence, 5'-3' } \\
\hline \multicolumn{6}{|l|}{ miR-1 } \\
\hline Forward & \multicolumn{5}{|c|}{ TGCTGATCCTTAGCAACCACACGGGTGTTTTGGCCACTGACTGACACCCGTGTTTGCTAAGGAT } \\
\hline Reverse & \multicolumn{5}{|c|}{ CCTGATCCTTAGCAAACACGGGTGTCAGTCAGTGGCCAAAACACCCGTGTGGTTGCTAAGGATC } \\
\hline \multicolumn{6}{|l|}{ miR-2 } \\
\hline Forward & \multicolumn{5}{|c|}{ TGCTGAGCTTCAGCCCATCCTTAGCAGTTTTGGCCACTGACTGACTGCTAAGGGGGCTGAAGCT } \\
\hline Reverse & \multicolumn{5}{|c|}{ CCTGAGCTTCAGCCCCCTTAGCAGTCAGTCAGTGGCCAAAACTGCTAAGGATGGGCTGAAGCTC } \\
\hline \multicolumn{6}{|l|}{ Control } \\
\hline Forward & \multirow{2}{*}{\multicolumn{5}{|c|}{$\begin{array}{l}\text { TGCTGGCATCATGACGTCGTGACCTACTCAGTAGATGCTCTAGTTAGTAATCTGATTGCGCACA } \\
\text { CCTGTGTGCGCAATCAGATTACTAACTAGAGCATCTACTGAGTAGGTCACGACGTCATGATGCC }\end{array}$}} \\
\hline Reverse & & & & & \\
\hline \multicolumn{6}{|l|}{ PTTG1 } \\
\hline Forward & \multicolumn{5}{|c|}{ CTGTAAAGACCAAGGGACCCCT } \\
\hline Reverse & \multicolumn{5}{|c|}{ GCAGGAACAGAGCTTTTTGCTT } \\
\hline \multicolumn{6}{|l|}{ GAPDH } \\
\hline Forward & \multicolumn{5}{|c|}{ GACAACTTTGGTATCGTGGAAGG } \\
\hline Reverse & \multicolumn{5}{|c|}{ CCAGTAGAGGCAGGGATGATGT } \\
\hline \multicolumn{6}{|c|}{$\begin{array}{l}\text { No other human messenger RNAs were observed to be homologous with the miR-1 or miR-2 sequences using the Basic Local Alignment Search } \\
\text { Tool in GeneBank. miR, microRNA; PTTG1, pituitary tumor-transforming gene 1; GAPDH, glyceraldehyde-3-phosphate dehydrogenase. }\end{array}$} \\
\hline \multicolumn{6}{|c|}{ Table II. Light absorption values (A490) of human malignant glioma U251 cells following transfection with miR-2 plasmids. } \\
\hline \multirow[b]{2}{*}{ Time point, $\mathrm{h}$} & \multicolumn{3}{|c|}{ Group } & \multirow[b]{2}{*}{ F-value } & \multirow[b]{2}{*}{ P-value } \\
\hline & Empty & Negative & $\operatorname{miR}-2$ & & \\
\hline 24 & $0.28 \pm 0.05$ & $0.27 \pm 0.02$ & $0.25 \pm 0.03$ & 7.0 & $<0.050$ \\
\hline 48 & $0.43 \pm 0.04$ & $0.39 \pm 0.04$ & $0.33 \pm 0.02$ & 76.0 & $<0.001$ \\
\hline 72 & $0.75 \pm 0.08$ & $0.72 \pm 0.06$ & $0.49 \pm 0.03^{\mathrm{a}}$ & 303.5 & $<0.001$ \\
\hline 96 & $0.97 \pm 0.10$ & $0.93 \pm 0.09$ & $0.68 \pm 0.07^{\mathrm{a}}$ & 741.0 & $<0.001$ \\
\hline
\end{tabular}

${ }^{a} \mathrm{P}<0.05$ vs. empty group. miR, microRNA.

glioma invasion and tumor angiogenesis, which requires additional investigation.

To study the effect of targeted silencing of the PTTG1 gene on the apoptotic rate of cells and the cell cycle in human malignant glioma U251 cells, the present study used Annexin V staining to detect cell apoptosis, while PI staining was used for cell cycle phase identification. Flow cytometry results suggested significant U251 cell apoptosis following miRNA transfection. Furthermore, the cells arrested at phase G2/M in the cell cycle, suggesting that there was significant cell apoptosis and cell cycle arrest following targeted suppression of PTTG1. These results are consistent with previous studies in other tumor cell lines $(19,20)$. The possible mechanism of PTTG1 in the process of cell apoptosis may be associated with the inhibitory effects of PTTG1 on apoptotic factors, including p53 and caspase family members, such as caspase 3 and caspase 7 (21). However, the elucidation of the detailed mechanism and demonstration of similar results to the study is required. Caspase 3 has been reported to be associated with glioma cell apoptosis. The cytotoxic cytokine cluster of differentiation 95 (22), tamoxifen (23) and adenovirus-mediated transfer of caspase 3 (24) greatly induced apoptosis of glioma cells in combination with intracellular $\mathrm{Ca}^{2+}$ homeostasis (25). The upstream regulator of caspase 3 has been associated with lipid peroxidation (26) and the Src-MAPK signaling pathway (27). Direct evidence was obtained in a previous RNA interference study that targeted the caspase 3 gene, which consequently suppressed the levels of apoptosis of human glioma cells (28). Furthermore, the gliomagenesis-associated miR-21 is known to exert its role via the inhibition of caspase 3 expression (29). The synergistic function of caspase 3 and p53 in etoposide-induced apoptosis has also been reported (30). Overall, these results clearly support the role of caspase 3 in the proliferation and apoptosis of glioma cells.

The present study demonstrated the essential role of PTTG1 in glioma cell proliferation and invasion, which was supported by the inhibition of PTTG1 expression and the corresponding suppression of U251 cell proliferation and 
migration. Furthermore, a targeted suppression of PTTG1 by exogenous miRNAs effectively induced U251 cell apoptosis and cell cycle arrest. Additional studies are required to investigate the role of PTTG1 as a regulator of p53 function, which may provide novel strategies for the treatment of malignant tumors.

\section{Acknowledgements}

The present study was supported by the National Natural Science Foundation of Jiangsu province (grant no. BK20130386), Jiangsu Planned Projects for Postdoctoral Research Funds (grant no. 1402200C) and Six Major Human Resources Project of Jiangsu Province (grant no. 2014-WSW-028).

\section{References}

1. Pei L and Melmed S: Isolation and characterization of a pituitary tumor-transforming gene (PTTG). Mol Endocrinol 11: 433-441, 1997.

2. Zhang X, Horwitz GA, Prezant TR, Valentini A, Nakashima M, Bronstein MD and Melmed S: Structure, expression, and function of human pituitary tumor-transforming gene (PTTG). Mol Endocrinol 13: 156-166, 1999.

3. Puri R, Tousson A, Chen L and Kakar SS: Molecular cloning of pituitary tumor transforming gene 1 from ovarian tumors and its expression in tumors. Cancer Lett 163: 131-139, 2001.

4. KakarSS,Chen L,Puri R,Flynn SEand Jennes L: Characterization of a polyclonal antibody to human pituitary tumor transforming gene 1 (PTTG1) protein. J Histochem Cytochem 49: 1537-1546, 2001.

5. Chamaon K, Kirches E, Kanakis D, Braeuninger S, Dietzmann K and Mawrin C: Regulation of the pituitary tumor transforming gene by insulin-like-growth factor-I and insulin differs between malignant and non-neoplastic astrocytes. Biochem Biophys Res Commun 331: 86-92, 2005

6. Vlotides G, Cruz-Soto M, Rubinek T, Eigler T, Auernhammer CJ and Melmed S: Mechanisms for growth factor-induced pituitary tumor transforming gene-1 expression in pituitary folliculostellate TtT/GF cells. Mol Endocrinol 20: 3321-3335, 2006.

7. Lewy GD, Ryan GA, Read ML, Fong JC, Poole V, Seed RI, Sharma N, Smith VE, Kwan PP, Stewart SL, et al: Regulation of pituitary tumor transforming gene (PTTG) expression and phosphorylation in thyroid cells. Endocrinology 154: 4408-4422, 2013.

8. Pei L: Activation of mitogen-activated protein kinase cascade regulates pituitary tumor-transforming gene transactivation function. J Biol Chem 275: 31191-31198, 2000.

9. Solbach C, Roller M, Peters S, Nicoletti M, Kaufmann M and Knecht R: Pituitary tumor-transforming gene (PTTG): A novel target for anti-tumor therapy. Anticancer Res 25: 121-125, 2005.

10. Qin B, Yang H and Xiao B: Role of microRNAs in endothelial inflammation and senescence. Mol Biol Rep 39: 4509-4518, 2012.

11. Saito Y and Jones PA: Epigenetic activation of tumor suppressor microRNAs in human cancer cells. Cell Cycle 5: 2220-2222, 2006.

12. Lewis BP, Burge CB and Bartel DP: Conserved seed pairing, often flanked by adenosines, indicates that thousands of human genes are microRNA targets. Cell 120: 15-20, 2005.

13. Livak and Schmittgen: Analysis of relative gene expression data using real-time quantitative PCR and the $2-\Delta \Delta \mathrm{Ct}$ method. Methods 25: 402-408, 2001.
14. Burger M, Hartmann T, Burger JA and Schraufstatter I: KSHV-GPCR and CXCR2 transforming capacity and angiogenic responses are mediated through a JAK2-STAT3-dependent pathway. Oncogene 24: 2067-2075, 2005.

15. Xia L, Huang Q, Nie D, Shi J, Gong M, Wu B, Gong P, Zhao L, Zuo H, Ju S, et al: PAX3 is overexpressed in human glioblastomas and critically regulates the tumorigenicity of glioma cells. Brain Res 1521: 68-78, 2013.

16. Lettau I, Hattermann K, Held-Feindt J, Brauer R, Sedlacek R and Mentlein R. Matrix metalloproteinase-19 is highly expressed in astroglial tumors and promotes invasion of glioma cells. J Neuropathol Exp Neurol 69: 215-223, 2010.

17. Heaney AP, Singson R, McCabe CJ, Nelson V, Nakashima M and Melmed S: Expression of pituitary-tumour transforming gene in colorectal tumours. Lancet 355: 716-719, 2000

18. Horwitz GA, Miklovsky I, Heaney AP, Ren SG and Melmed S: Human pituitary tumor-transforming gene (PTTG1) motif suppresses prolactin expression. Mol Endocrinol 17: 600-609, 2003.

19. Mora-Santos M, Castilla C, Herrero-Ruiz J, Giráldez S, Limón-Mortés MC, Sáez C, Japón MÁ, Tortolero M and Romero F: A single mutation in Securin induces chromosomal instability and enhances cell invasion. Eur J Cancer 49: 500-510, 2013.

20. Huang SQ, Liao QJ, Wang XW, Xin DQ, Chen SX, Wu QJ and Ye G: RNAi-mediated knockdown of pituitary tumor-transforming gene-1 (PTTG1) suppresses the proliferation and invasive potential of PC3 human prostate cancer cells. Braz J Med Biol Res 45: 995-1001, 2012.

21. Kim D, Pemberton H, Stratford AL, Buelaert K, Watkinson JC, Lopes V, Franklyn JA and McCabe CJ: Pituitary tumour transforming gene (PTTG) induces genetic instability in thyroid cells. Oncogene 24: 4861-4866, 2005.

22. Hueber A, Winter S and Weller M: Chemotherapy primes malignant glioma cells for CD95 ligand-induced apoptosis up-stream of caspase 3 activation. Eur J Pharmacol 352: 111-115, 1998.

23. Tseng SH, Wang CH, Lin SM, Chen CK, Huang HY and Chen Y: Activation of c-Jun N-terminal kinase 1 and caspase 3 in the tamoxifen-induced apoptosis of rat glioma cells. J Cancer Res Clin Oncol 130: 285-293, 2004.

24. Shinoura N, Muramatsu Y, Yoshida Y, Asai A, Kirino T and Hamada H: Adenovirus-mediated transfer of caspase-3 with Fas ligand induces drastic apoptosis in U-373MG glioma cells. Exp Cell Res 256: 423-433, 2000.

25. Qi H, Chen HZ and Jin ZJ: Caspase 3 gene expression and [Ca2+]i homeostasis underlying desipramine-induced C6 glioma cell apoptosis. Acta Pharmacol Sin 23: 803-807, 2002.

26. Higuchi Y and Yoshimoto T: Arachidonic acid converts the glutathione depletion-induced apoptosis to necrosis by promoting lipid peroxidation and reducing caspase-3 activity in rat glioma cells. Arch Biochem Biophys 400: 133-140, 2002.

27. Beretta F, Bassani S, Binda E, Verpelli C, Bello L, Galli R and Passafaro M: The GluR2 subunit inhibits proliferation by inactivating Src-MAPK signalling and induces apoptosis by means of caspase 3/6-dependent activation in glioma cells. Eur J Neurosci 30: 25-34, 2009.

28. Kugler W, Buchholz F, Köhler F, Eibl H, Lakomek M and Erdlenbruch B: Downregulation of Apaf-1 and caspase-3 by RNA interference in human glioma cells: Consequences for erucylphosphocholine-induced apoptosis. Apoptosis 10: 1163-1174, 2005 .

29. Zhou X, Zhang J, Jia Q, Ren Y, Wang Y, Shi L, Liu N, Wang G, $\mathrm{Pu}$ P, You Y and Kang C: Reduction of miR-21 induces glioma cell apoptosis via activating caspase 9 and 3. Oncol Rep 24: 195-201, 2010.

30. Yin D, Tamaki N and Kokunai T: Wild-type p53-dependent etoposide-induced apoptosis mediated by caspase-3 activation in human glioma cells. J Neurosurg 93: 289-297, 2000. 